\title{
Limitações do paradigma científico hegemônico no acolhimento ao sofrimento psíquico na atenção básica ${ }^{*}$
}

\author{
Maria Luisa Vichi de Campos Faria ${ }^{1}$
}

Ivan Amaral Guerrini ${ }^{2}$

CAMPOS FARIA, M.L.V.; GUERRINI, I.A. Limitations of the hegemonic scientific paradigm for dealing with mental distress at primary healthcare units. Interface Comunic., Saude, Educ., v.16, n.42, p.779-92, jul./set. 2012.

The problem that motivated the present study was the difficulty in dealing with mental distress at primary healthcare units. In this study, we investigated whether this difficulty was due to epistemological deficiencies of the hegemonic paradigm. We reviewed the literature and analyzed the use of the term paradigm in recent Brazilian scientific articles. After searching using the keywords paradigm and medicine and making a preselection, 17 texts were chosen. The analysis was done in two stages: linguistic analysis and surveying of emerging themes. We surveyed the fifty most commonly used words and grouped them into communities of cooccurrence using the Clauset algorithm. The texts discussed Kuhn's ideas and presented innovative experiences already implemented in healthcare units and examples of curricular changes in medicine courses. Health promotion appeared as a new and promising paradigm for healthcare.

Keywords: Paradigm. Medicine. Mental distress. Primary care. Health promotion.
O problema que motivou a realização do presente trabalho foi a dificuldade em se acolher o sofrimento psíquico nas unidades de atenção básica. Neste trabalho, investigamos se esta dificuldade se deve à inadequação epistemológica do paradigma hegemônico. Realizamos uma revisão de literatura e analisamos a utilização do termo paradigma na produção científica brasileira recente. Após busca pelas palavras-chave paradigma e medicina e seleção prévia, foram escolhidos 17 textos. A análise foi feita em duas etapas: análise linguística e levantamento da temática emergente. Levantamos as cinquenta palavras mais frequentes e as agrupamos em comunidades de coocorrência, utilizando o algoritmo de Clauset. Os textos discutiam as ideias de Kuhn e trouxeram tanto experiências inovadoras já implantadas em unidades de saúde quanto exemplos de mudanças curriculares nos cursos de medicina. A promoção da saúde aparece como um novo e promissor paradigma para a saúde.

Palavras-chave: Paradigma. Medicina. Sofrimento psíquico. Atenção primária à saúde. Promoção da saúde.
* Elaborado com base em Campos Faria (2009).

1 Equipe de Matriciamento em Saúde Mental, Secretaria Municipal de Saúde de Botucatu. Rua João de Oliveira, 1017, Altos do

Paraíso. Botucatu, SP

Brasil. 18.610-010.

ml.vichi@gmail.com

${ }^{2}$ Departamento de Física e Biofísica, Instituto de Biociências de Botucatu,

Universidade Estadua Paulista Júlio de Mesquita Filho. 


\section{Introdução}

O conceito de paradigma proposto por Thomas Kuhn (1922-1996) foi discutido no livro "A estrutura das revoluções científicas", publicado pela primeira vez em 1962. Essa obra teve versões em 25 idiomas, sendo que a versão em inglês vendeu mais de um milhão de exemplares. O número de livros vendidos e, também, o debate fomentado a partir desse autor demonstram sua importância para as discussões sobre ciência ao redor do mundo. Ao consultar registros históricos de atividades de pesquisa, Kuhn questionou o dogma vigente de que o progresso da ciência se dava por acúmulo de conhecimento. Ele passou, então, a defender sua ideia de que a ciência progredia não por acúmulo de conhecimento, mas por processos contraditórios, descontínuos e em saltos qualitativos marcados pelas revoluções científicas.

Kuhn define que "paradigma é aquilo que os membros de uma comunidade partilham e, inversamente, uma comunidade científica consiste em homens que partilham um paradigma" (Kuhn, 1970, p.221). É a existência de um paradigma que coloca o problema a ser resolvido e concebe instrumentos capazes de resolvê-lo, sendo o papel da ciência normal a sua consolidação. A ciência normal, segundo Kuhn (1970), é uma forma de pesquisa que tenta vigorosa e devotadamente forçar a natureza aos esquemas conceituais aprendidos na educação profissional, e está baseada no pressuposto de que a comunidade científica sabe como o mundo funciona.

A ciência normal se desorganiza seguidamente em função das anomalias que têm como traço característico a sua recusa obstinada de serem assimiladas pelo paradigma vigente. A consciência da anomalia se dá pelo reconhecimento de que, de alguma maneira, a natureza violou as expectativas paradigmáticas da ciência normal ou quando surge um fenômeno para o qual o paradigma não havia preparado o profissional. Quando os pesquisadores não podem mais se esquivar das anomalias, iniciamse, então, as investigações extraordinárias, que, finalmente, os conduzem a um novo conjunto de compromissos e a uma nova base para a prática da ciência. Estes episódios extraordinários, nos quais ocorre a substituição dos antigos compromissos por novos, são denominados de revoluções científicas. A transição de um paradigma em crise para o novo é uma reconstrução da área de estudos a partir de novos princípios que alterarão algumas das generalizações teóricas mais elementares do paradigma, bem como muitos de seus métodos, aplicações e instrumentos (Kuhn, 1970).

Thomas Kuhn considera também que paradigmas são "as realizações científicas universalmente reconhecidas que, durante algum tempo, fornecem problemas e soluções modelares para uma comunidade de praticantes de uma ciência" (Kuhn, 1970, p. 13). Um paradigma adquire seu status porque se mostra mais competente que outro na solução de problemas que um grupo de cientistas identificou como importantes. Decidir rejeitar um paradigma é sempre decidir aceitar simultaneamente outro e, para tanto, se faz necessária uma comparação entre o paradigma vigente e o novo quanto à sua capacidade em lidar com a natureza do problema a ser enfrentado (Kuhn, 1970).

$\mathrm{Na}$ área da saúde, diversos autores vêm questionando a eficiência do paradigma hegemônico para o enfrentamento dos problemas de saúde no mundo, sua adequação para definição de políticas públicas, na formação dos médicos e no modelo de assistência. A crise na saúde não é uma questão meramente política que, como tal, poderia ser equacionada com vontade política e decisões técnico-administrativas. É necessário ver todas as dimensões do problema e, sobretudo, examinar as dificuldades produzidas por obstáculos internos à própria racionalidade biomédica (Guedes, Nogueira, Camargo Jr., 2006).

Valla (2001) cunhou o termo "sofrimento difuso", que tem sido definido como um tipo de sofrimento psíquico mais leve, caracterizado pela presença de múltiplos sintomas que se expressam por meio de: queixas somáticas inespecíficas, dores generalizadas, medo, ansiedade, insônia, nervosismo, baixa autoestima e perda da esperança de vida. O sofrimento difuso se enquadra dentro do que a epidemiologia denomina de Transtornos Mentais Comuns (TMCs) (Guimarães, 2007). Os TMCs se enquadram na situação de saúde de uma população com indivíduos que não preenchem os critérios formais para diagnósticos de depressão e/ou ansiedade segundo as classificações da quarta edição do Diagnostic and Statistical Manual of Mental Disorders (DSM-IV) e a décima revisão da Classificação Internacional de Doenças (CID-10), mas que apresentam sintomas proeminentes que trazem uma 
incapacitação funcional comparável ou até pior do que quadros crônicos já bem estabelecidos (Santos, 2002). Levando-se em conta que a prevalência dos TMCs, segundo estudos em atenção primária no Brasil, gira em torno de $56 \%$ (Fortes, Villano, Lopes, 2008), percebe-se a magnitude do problema causado pelo não-acolhimento do sofrimento psíquico na atenção básica.

Segundo o Ministério da Saúde (Brasil, 2003), os TMCs parecem ser responsáveis por uma parcela significativa da demanda por consultas médicas, já que $56 \%$ das equipes de saúde da família relataram a realização de alguma ação em saúde mental. Dessa forma, percebe-se que o não-acolhimento a essa demanda gera problemas tanto para as unidades básicas, que não conseguem agendamentos necessários para os profissionais "psi", quanto para os serviços especializados já sobrecarregados. Geram-se, ainda, problemas para os gestores, que nunca terão especialistas suficientes, e, também, para os indivíduos em sofrimento, que terão de enfrentar uma longa espera pelas consultas com os especialistas.

Nesse artigo, utilizamos uma metodologia inspirada na análise de conteúdo (Bardin, 1977) em novas metodologias oriundas das áreas de processamento de linguagem natural e teoria das redes complexas (Barabási, Gulbahce, Loscalzo, 2011). Com base nessas metodologias, foram selecionadas as palavras mais frequentes e seus inter-relacionamentos encontrados numa seleção de 17 textos. Em seguida, foi levantada a temática emergente e realizado um levantamento sobre as bases teóricas utilizadas pelos autores dos textos. Na discussão dos resultados, verificaremos se os mesmos contribuem para que possamos responder à pergunta motivadora desse trabalho: seria o sofrimento psíquico uma anomalia para o paradigma hegemônico da medicina?

\section{Metodologia}

A fonte utilizada para essa revisão bibliográfica foi o SciELO-br, com o cruzamento das palavraschave "paradigma" e "medicina". Nessa busca, foram encontrados oitenta textos, que tiveram seus resumos lidos e, a partir dessa leitura, foram selecionados trinta textos baseados nos seguintes critérios: ter sido publicado entre 1998 e 2008, apresentar uma análise crítica do paradigma médico hegemônico e discutir novos paradigmas para a prática médica na atenção básica em saúde. Os trinta textos foram lidos na íntegra, sendo, então, excluídos 13 textos por não se enquadrarem nos critérios acima citados.

Segundo Bardin (1977), a análise de conteúdo (AC) é um conjunto de instrumentos metodológicos em constante aperfeiçoamento, que se aplicam a "discursos" (conteúdos e continentes) extremamente diversificados. Não há uma regra geral em AC, mas, sim, algumas regras de base; por esse motivo, a AC é uma técnica que requer constante reinvenção de acordo com o domínio e o objetivo pretendidos. Enquanto esforço de interpretação, a AC oscila entre o rigor da objetividade e a fecundidade da subjetividade, sendo a superação da incerteza e o enriquecimento da leitura seus principais objetivos.

A partir da década de 1960, o uso do computador trouxe vantagens para a AC por permitir rápida assimilação de maior quantidade de dados e acréscimo de rigor às análises. Com a ajuda desse recurso, as técnicas de análise puderam ser orientadas não somente para a simples frequência da aparição dos elementos do texto, mas também para as relações entre esses elementos. A técnica de análise das coocorrências ou análise de contingências (contingency analysis), esboçada por Baldwin em 1942 e aperfeiçoada por Osgood em 1959, complementou a análise frequencial simples (Bardin, 1977).

A análise das coocorrências procura extrair dos textos as relações entre os elementos da mensagem ou, mais exatamente, dedica-se a assinalar as presenças simultâneas (coocorrência ou relação de associação) de dois ou mais elementos na mesma unidade de contexto, isto é, num fragmento de mensagem previamente definido. Enquanto a frequência de aparição das unidades de significação ou de elementos formais assenta-se no princípio de que quanto maior for a frequência dos elementos, tanto maior será a sua importância, a coocorrência de dois ou mais elementos revelaria a associação entre eles na mente do locutor (Bardin, 1977).

As etapas do procedimento de Osgood são (Bardin, 1977, p.192): 
1 Escolha das unidades de registro (palavras-chave, por exemplo) e a categorização (temas), se tal tiver cabimento.

2 Escolha das unidades de contexto e a decomposição do texto em fragmentos. Osgood sugere que a unidade de contexto seja constituída por fragmentos de 120 a 210 palavras para que as coocorrências tenham sentido.

3 Codificação: presença ou ausência de cada unidade de registro (elemento) em cada unidade do contexto.

4 Cálculo das coocorrências (matriz de contingência).

Para Osgood, esse tipo de análise é apropriado para as mensagens espontâneas, não estratégicas e, também, para mensagens deliberadas com origem institucional. A análise de contingências pode ser utilizada para clarificar as estruturas da personalidade, as "preocupações latentes" individuais ou coletivas, os estereótipos, as representações sociais e as ideologias, já que o locutor (ou grupos de locutores) não pode controlar totalmente as suas associações, assim falsificando o sentido das coocorrências manifestadas no texto (Bardin, 1977).

A AC foi feita em duas etapas: análise linguística, com análises de frequência e coocorrência, e levantamento da temática emergente.

Foi realizada a análise de frequência das palavras do conjunto dos 17 textos escolhidos, tomando-se o cuidado de excluir artigos, verbo "ser" em seus diferentes tempos verbais e outras palavras sem relevância para o tema do artigo. Em seguida, foram tomadas as cinquenta palavras mais citadas e determinou-se o número de vezes que estas palavras apareciam próximas no texto (vinte palavras de distância), visando, com isso, descartar aparições fortuitas. Consideramos que duas palavras estavam correlacionadas se elas estivessem próximas ao menos dez vezes.

Finalmente, agrupamos as palavras em comunidades, utilizando o algoritmo de Clauset (Clauset, Newman, Moore, 2004). Estas análises foram realizadas com a utilização do aplicativo Mathematica ${ }^{\circledR}$ 7.0, através de pacotes desenvolvidos para este propósito por um especialista na área de informática.

\section{Apresentação dos resultados}

O objeto de estudo deste trabalho se constituiu do conjunto dos 17 textos abaixo enumerados, segundo ordem cronológica do ano de publicação (dados completos na lista de referências, ao final do manuscrito):

1 PAIM, J. S.; ALMEIDA FILHO, N. Saúde coletiva: uma "nova saúde pública" ou campo aberto a novos paradigmas?

2 MARTINS, A. Novos paradigmas e saúde.

3 CARDOSO, M.H.C.A. História e medicina: a herança arcaica de um paradigma.

4 JACOBINA, R.R. O paradigma da epistemologia histórica: a contribuição de Thomas Kuhn.

5 QUEIROZ, M.S. O itinerário rumo às medicinas alternativas: uma análise em representações sociais de profissionais da saúde.

6 TESSER, C.D.; LUZ, M.T. Uma introdução às contribuições da epistemologia contemporânea para a medicina.

7 FREITAS, C.M. Problemas ambientais, saúde coletiva e ciências sociais.

8 AYRES, J.R. Norma e formação: horizontes filosóficos para as práticas de avaliação no contexto da promoção da saúde.

9 LUZ, M.T. Cultura contemporânea e medicinas alternativas: novos paradigmas em saúde no fim do século $X X$.

10 SOUSA, I.M.C.; VIEIRA, A.L.S. Serviços públicos de saúde e medicina alternativa.

11 RIBEIRO, J.A.S. et al. Promoção da saúde e cultura cidadã envolvendo uma abordagem oftalmológica em escolares na Colônia Antônio Aleixo (CAA), Manaus-AM: uma experiência no ensino médico. 
12 NOGUEIRA, M.I.; CAMARGO JR., K.R. A orientalização do Ocidente como superfície de emergência de novos paradigmas em saúde.

13 PEREIRA, M.A.O. et al. Saúde mental no Programa de Saúde da Família: conceitos dos agentes comunitários sobre o transtorno mental.

14 TESSER, C.D. Contribuições das epistemologias de Kuhn e Fleck para a reforma do ensino médico.

15 ARAÚJO, E.C. Homeopatia: uma abordagem do sujeito no processo de adoecimento.

16 GALHARDI, W.M.P.; BARROS, N.F. O ensino da homeopatia e a prática no SUS.

17 ASSUNÇÃO, L.F.; MELO, G.C.M.P.; MACIEL, D. Relação médico-paciente permeando o currículo na ótica do estudante.

\section{Análise de frequência}

Na Figura 1, são apresentadas as palavras mais utilizadas nos textos analisados. A frequência é um indicador da relevância de um termo em uma determinada área de investigação. As palavras que apareceram mais de duzentas vezes nos textos estão listadas na Tabela 1, onde apresentamos seus principais contextos.

Figura 1. Palavras com maior ocorrência nos textos estudados; algumas palavras sem relevância semântica foram excluídas (artigos, verbos ter e ser etc.)

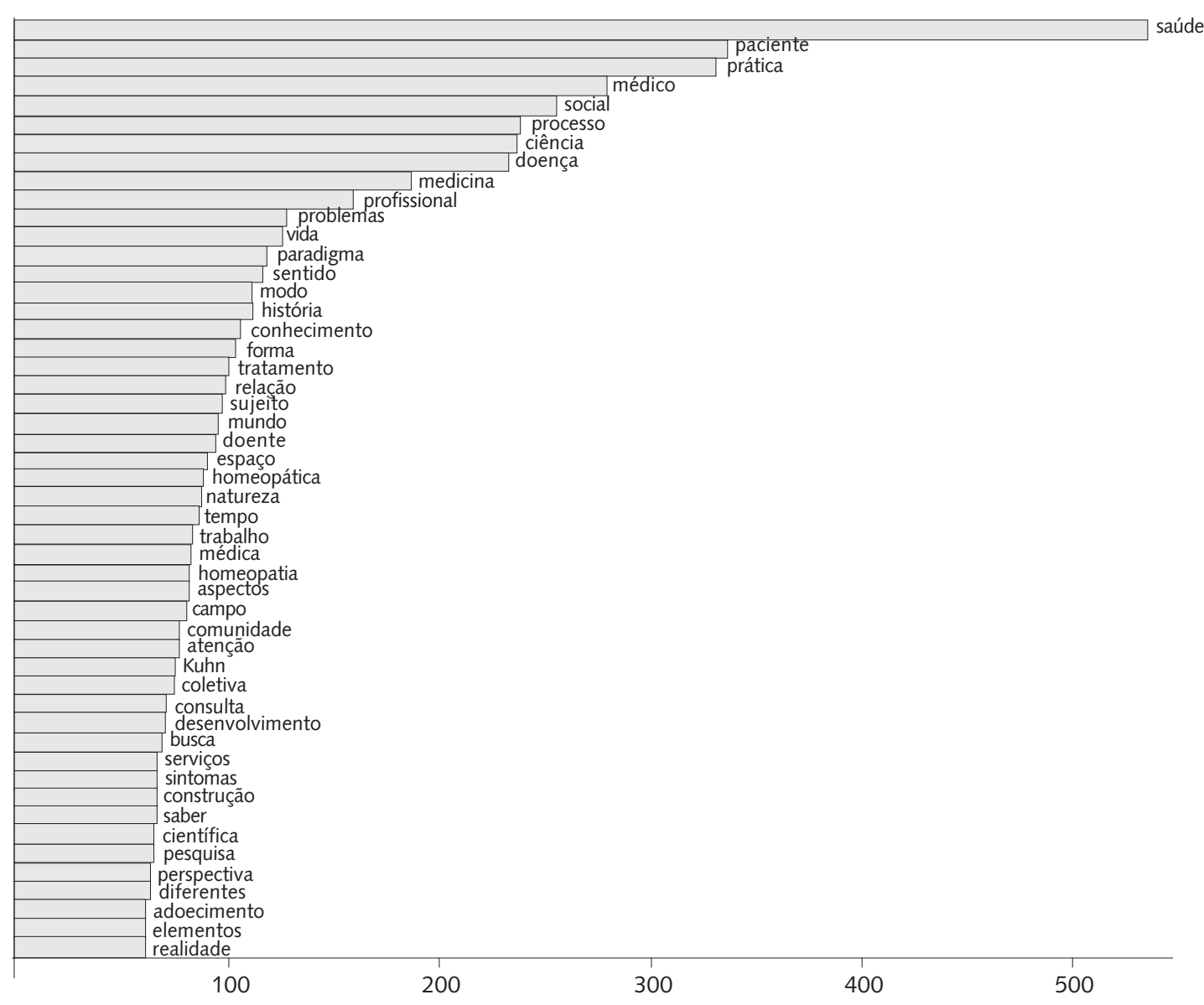


Tabela 1. Palavras mais usadas e os principais contextos em que são utilizadas

\begin{tabular}{ll}
\hline Palavra & \multicolumn{1}{c}{ Discussão de Contexto } \\
\hline Saúde & Novo entendimento sobre sua definição \\
& Foco do paradigma na saúde, e não na doença \\
& Paradigma da promoção da saúde é citado pela maioria dos artigos \\
Prática & Críticas à prática médica no modelo hegemônico \\
& Necessidade de práticas alternativas de tratamento \\
& Integração do estudo teórico à prática na comunidade precocemente \\
Social & Dimensão social da doença e da saúde \\
& Noção de saúde social \\
Paciente & Foco do paradigma no paciente, e não na doença \\
& Importância da relação médico-paciente \\
Ciência & Visão moderna do paciente cidadão \\
Médico & Influência da ciência no ensino médico e atividade médica \\
& Relação médico-paciente \\
& Críticas e sugestões sobre o ensino médico \\
& Papel simbólico do médico \\
Conflitos entre o médico e paciente \\
Processo & Habilidades exigidas do médico contemporâneo \\
& Críticas ao paradigma focado na doença \\
& Novas noções sobre doença \\
& Processo saúde-doença diferente do binômio saúde-doença \\
& Adoecimento como um processo diferente do binômio saúde-doença \\
\end{tabular}

Com base na lista das palavras mais utilizadas, foi construído um grafo de coocorrências, onde se visualizam os temas e suas relações, apresentadas na Figura 2. Os temas foram organizados em comunidades para facilitar a visualização. Na comunidade 1, estão os termos centrais nos textos avaliados, como saúde, prática, vida etc., e termos das ciências sociais, como social, comunidade, trabalho e coletiva. A comunidade 2 reúne termos ligados à medicina alternativa (homeopatia e homeopática). A comunidade 4 apresenta alguns termos ligados à epistemologia e à filosofia da ciência, como Kuhn e pesquisa. O termo paradigma não é um termo especialmente citado apesar de estar na comunidade 1. O termo doente forma uma comunidade isolada, mostrando que a palavra é usada em vários contextos.

Os termos paradigma, conhecimento e ciência aparecem juntamente com outros termos sociológicos. Isto reflete que a obra de Kuhn apresenta a epistemologia dentro de uma visão sociológica do fazer científico, sendo este aspecto ressaltado nos textos aqui discutidos. É natural que a medicina, sendo uma área de conhecimento aplicado, apareça impregnada de aspectos das ciências sociais mesmo numa discussão epistemológica. Este aspecto surge uma vez mais quando avaliamos os autores e seus referenciais teóricos (Figura 3).

Uma outra análise foi feita visando determinar as principais referências bibliográficas utilizadas pelos autores dos 17 textos. Inicialmente, foi feito um levantamento de todos os principais autores citados e, então, foram determinados quais trabalhos citavam estes autores principais. Não foi feita distinção por obra citada e, sim, por autores. Na Figura 3, constatamos que Kuhn e Foucault são fortemente citados, mas também autores ligados à sociologia da ciência, como Latour, Lakatos e Fleck. Minayo também é citada por atuar em áreas que envolvem a epistemologia, as ciências sociais e suas aplicações em saúde coletiva. Apesar de citado, Morin, que discute epistemologia da ciência e o paradigma da complexidade, foi pouco discutido. 
Figura 2. Grafo representando as coocorrências dos diferentes termos mais frequentes. Uma ligação é traçada sempre que as palavras são encontradas próximas nos diferentes textos. Os conjuntos indicam a organização em comunidades usando-se o algoritmo de Clauset (Clauset, Newman, Moore, 2004)

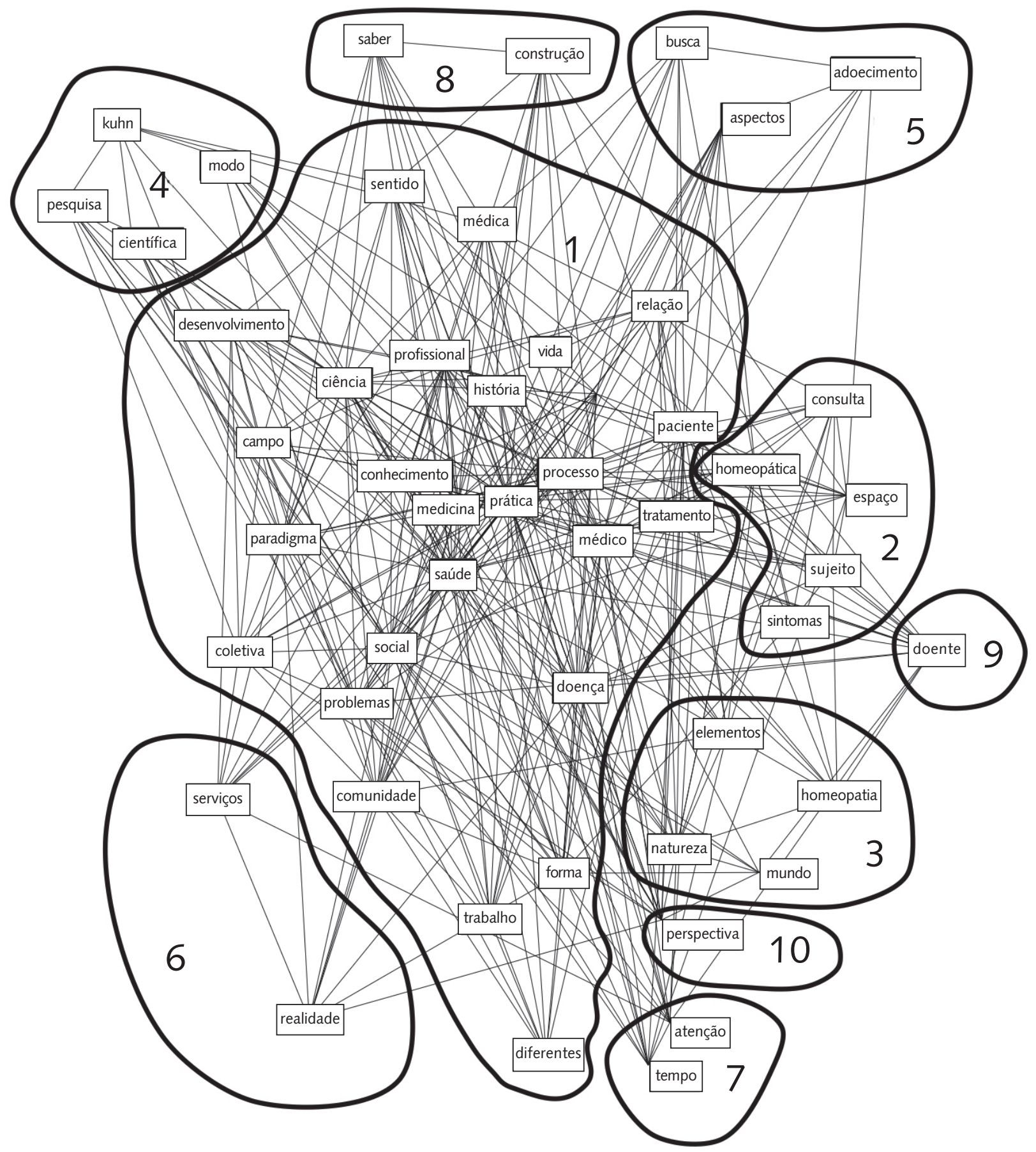


Figura 3. Grafo com os autores e seus referenciais teóricos. Para facilitar a visualização, os autores são representados apenas pelo sobrenome

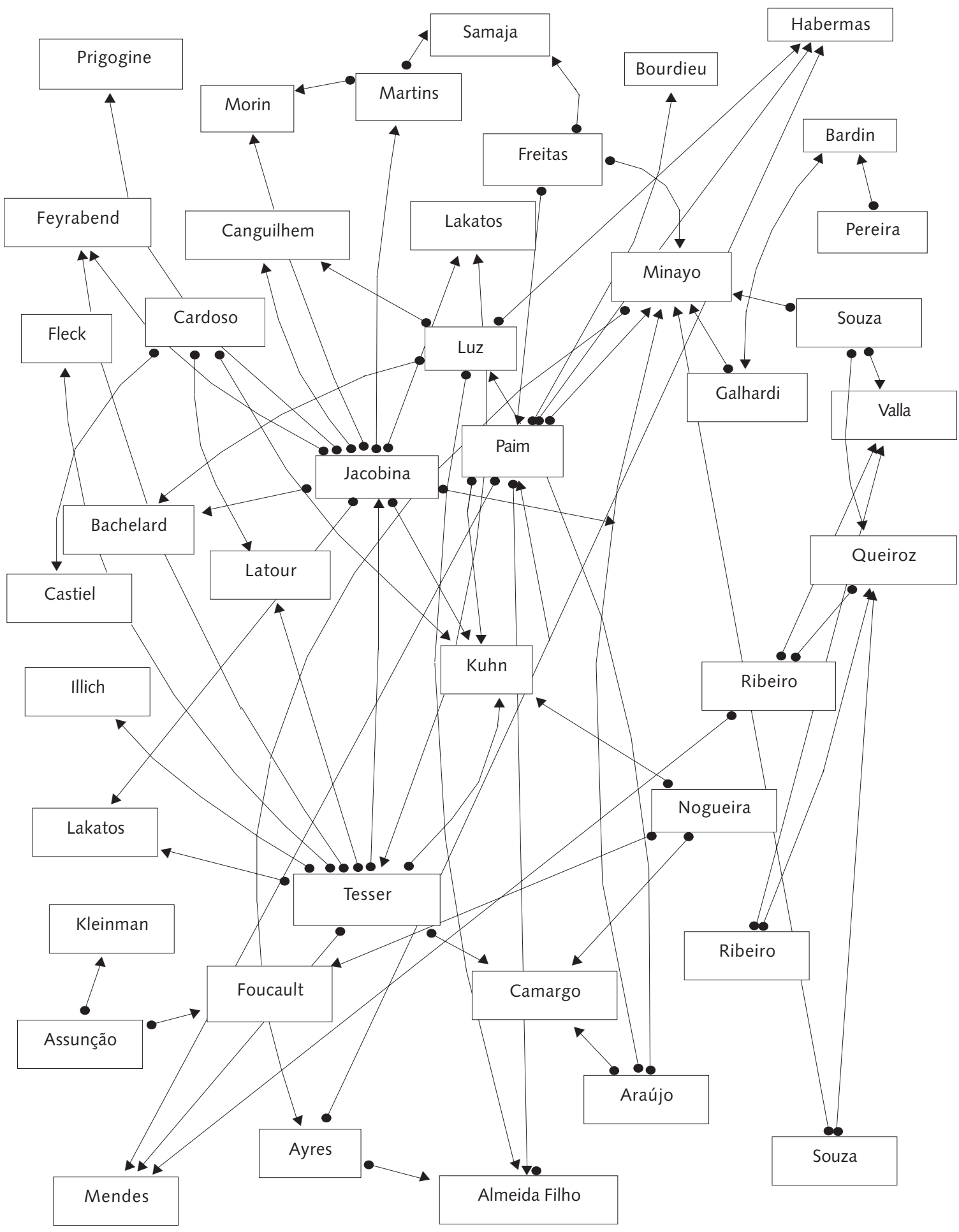




\section{Temática emergente}

Para o levantamento da temática emergente, os textos foram selecionados conforme seu foco principal em três grupos (Tabela 2).

A partir dos textos 1, 2, 3, 4, 6, 7, 9 e 12, emerge uma temática com foco teórico, na qual discutem-se: o papel central da doença no paradigma hegemônico, os sinais de crise na medicina e na saúde, a interface entre saúde e meio ambiente, e o modelo da promoção da saúde.

Uma temática centrada no ensino médico, por sua vez, emerge dos textos 11, 14, 16 e 17. Nessa temática, discutem-se a ambiência da formação do médico, novos temas curriculares, tais como: integralidade, humanização, qualidade de vida, relação médico-paciente, interdisciplinaridade, promoção da saúde, questões emocionais dos médicos e formação profissional crítica, e a qualificação dos professores dos cursos de medicina para ensinar fora dos hospitais e para discutir os novos temas curriculares. A partir dos textos remanescentes 5, 8, 10, 13 e 15, emerge a temática sobre a prática médica.

Nessa temática, discutem-se: o foco do paradigma hegemônico na doença, a intermediação tecnológica na prática da medicina, a integralidade e singularidade do doente e do cuidado, a relação médico-paciente como elemento terapêutico, e a promoção da saúde como novo e promissor paradigma da saúde.

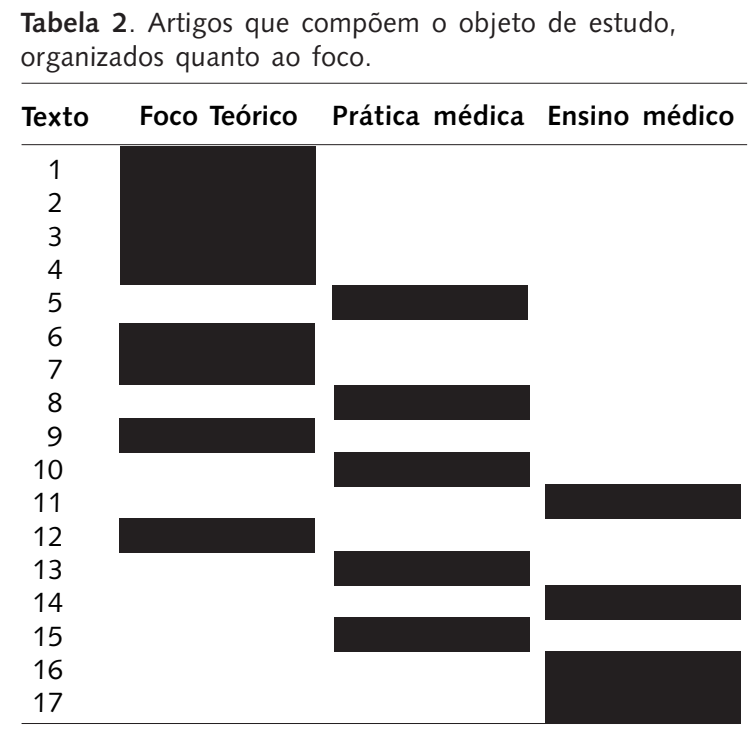

\section{Discussão dos resultados}

O paradigma que rege a medicina contemporânea tem como elementos constituintes: a fragmentação, o reducionismo, a especialização, a visão dualista do homem e o crescente foco no tecnicismo diagnóstico e terapêutico. Consequentemente, tal paradigma é limitado e, às vezes, ineficaz para o acolhimento do sofrimento psíquico, condição esta que, por demandar integralidade de atenção, respeito às singularidades e conhecimento das condições de vida do indivíduo, não se enquadrará, portanto, na "prática normal" de uma unidade básica de saúde cuja organização se dá numa lógica biomédica: triagem, consultas, procedimentos, encaminhamentos aos especialistas e palestras para os grupos. Os indivíduos, ao trazerem seu mal-estar e suas queixas não reconhecidas por esse paradigma, 
subvertem, dessa forma, os fluxos da unidade de saúde. O sofrimento psíquico, enquanto anomalia, não tem lugar nessas unidades, sendo, por isso, endereçado aos especialistas.

A "crise de harmonia clínica" e a crise da saúde pública podem ser importantes sinalizadores de que o modelo de formação profissional, os processos de trabalho e a organização das unidades básicas de saúde precisam ser norteados por princípios de um outro paradigma.

Seguem trechos dos textos selecionados que balizaram a discussão da tese central deste trabalho:

- Para Kuhn, todo paradigma orientador de uma fase normal do desenvolvimento de uma disciplina científica é pouco ou nada questionado internamente pelos seus praticantes (Tesser, Luz, 2002, p.366).

- Um ponto-chave no pensamento de Kuhn refere-se ao fato de que o paradigma científico é sustentado, basicamente, não só por sua consistência lógica e epistemológica, nem por sua adequação aos fatos, mas, sobretudo, pela comunidade científica e pela sociedade mais ampla, em termos que incluem interesses sociais, econômicos e culturais (Queiroz, 2000, p.367).

- Embora o conceito kuhniano tenha sido forjado numa perspectiva histórica, retrospectiva e restrita à produção do saber, a rigor, somente no futuro poderíamos caracterizar um paradigma para o saber da biomedicina dos séculos XX e XXI. Mesmo assim, tem se mostrado fértil e produtiva uma extensão desse conceito para o presente momento e para a prática médica, tornando possível uma descrição de algumas características centrais de tal paradigma (Tesser, 2008, p.3).

- A biomedicina surgiu entre os séculos XVI e XVII, trazendo as explicações mecanicistas dos fenômenos do adoecimento, a fragmentação do conhecimento em especialidades e uma visão dualista (corpo/alma) do homem. A medicina científica moderna tem concepção tecnicista e atomizante do homem e de suas doenças, concepção baseada na física clássica herdada de Newton e no dualismo do ser humano herdado de Descartes (Nogueira, Camargo Jr., 2007, p.859).

- O paradigma hegemônico da medicina é o biomecânico, centrado nas entidades doenças da nosografia biomédica. Em função do excesso de importância metodológica que converge para elas, as doenças passaram a dominar todo o proceder investigativo (metodológico), teórico e terapêutico da medicina. Há um esquartejamento epistemológico do doente em síndromes e doenças de aparelhos orgânicos (e especialistas), bem separados por uma fisiologia e fisiopatologia biomecânicas, que sabe muito de patologias, micro-organismos, moléculas, órgãos e tecidos do corpo, e muito pouco das ligações e inter-relações complexas entre esses componentes e a vida do doente (Tesser, Luz, 2002, p.367).

- Os médicos e demais profissionais de saúde passaram, progressivamente, a ser técnicos, tais como os cientistas aplicadores da ciência normal. Eles devem resolver um pequeno enigma a cada novo doente: qual a doença que o doente tem? Resolvido esse enigma, acabaram seus problemas e desafios, restando apenas aplicar a terapêutica padronizada em voga na atualidade científica (Tesser, Luz, 2002, p.367).

- No paradigma que rege a medicina contemporânea, o sujeito humano sofredor deixou de ser o centro de seu objeto (como investigação) e de seu objetivo (como prática terapêutica). A medicina ocidental distanciou-se da sua dimensão terapêutica, na busca de investigar, classificar e explicar antigas e novas patologias através de métodos diagnósticos sofisticados (Luz, 2005, p.151).

- A situação de crise e possível transição paradigmática apresenta-se de modo mais agudo, e com contornos próprios, nas áreas de conhecimento aplicado, como é o caso da saúde pública, saúde mental, medicina e psiquiatria, cujas práticas baseiam-se tanto nas ciências naturais quanto nas sociais (Jacobina, 2000, p.628).

- Vale lembrar que a crise da medicina não significa uma crise em seu modelo de produção de conhecimento: muito pelo contrário, em termos de ciência das doenças, a medicina vai muito bem, revolucionando-se constantemente através da produção dos ramos disciplinares da biociência (Luz, 2005, p.151).

- As modificações do panorama político e social do mundo e da situação de saúde (especialmente a falta de mudanças esperadas) põem em cheque as premissas e previsões dos antigos modelos. A partir da constatação do esgotamento dos paradigmas vigentes, refletidos pela crise da saúde pública, surgem demandas por novos paradigmas. Para um debate a esse respeito, é também necessário levar em consideração as restrições econômicas que comprometem a capacidade de o Estado suportar, a longo 
prazo, o custo crescente da atenção à saúde, particularmente a assistência médico-hospitalar, que não garante às populações melhor nível de bem-estar (Paim, Almeida Filho, 1998, p.12).

- Na prática diária da clínica biomédica, é muito frequente que doentes sejam encaminhados a vários especialistas por falta de diagnóstico, e é comum que se esgote o arsenal terapêutico sem resolubilidade clínica satisfatória. Pode-se chamar de crise de harmonia clínica o conjunto de dilemas e tensões que ocorrem cotidianamente nos atendimentos biomédicos nos serviços de saúde. Além disso, essa perspectiva reforça a ênfase no uso de tecnologia biomédica dura (materializada em equipamentos diagnósticos e terapêuticos interpostos entre o médico e o doente), obscurecendo a importância da relação médico-paciente, secundarizando a terapêutica em favor do diagnóstico e submetendo os médicos a um objetivismo excessivo. Dessa forma, os pacientes não enquadráveis seguem numa via crucis pelas especialidades médicas (Tesser, Luz, 2002, p.364).

- Uma parte significativa da demanda que busca atenção médica ambulatorial não preenche os requisitos para um diagnóstico de base anatomoclínica, pois suas queixas são vagas e inespecíficas, ou seja, manifestações de um sofrimento que não se traduz, necessariamente, em lesões ou disfunções que possam ser enquadradas em alguma nosologia (Araújo, 2008, p.664).

- Há uma tendência nos serviços de saúde à desqualificação das queixas de pacientes, traduzidas por mal-estares e sintomas indefinidos, gerando desencontros entre a expectativa do médico em identificar e reconhecer doenças e as demandas do doente de obter atenção para o seu sofrimento (Araújo, 2008, p.664).

- A redução paradigmática refere-se ao fato de que a abordagem de qualquer fenômeno orientada por um paradigma gera um saber que diz respeito ao fenômeno sempre de forma incompleta e parcial. Um paradigma seleciona partes da realidade e deixa outras partes e fenômenos, ou suas relações, de fora. Por vezes, tais fenômenos ou relações não previstos aparecem aos cientistas, constituindo o que Kuhn chamou de anomalias, como, por exemplo, a subjetividade dos doentes no paradigma da biomedicina (Tesser, 2008, p.6).

- O peso dos sintomas subjetivos é pouco valorizado para o diagnóstico porque os procedimentos tecnológicos prevalecem sobre eles (Galhardi, Barros, 2008). Nesse contexto, curas, sintomas ou sinais que sejam inexplicáveis, não previstos, ou contraditórios, pelas teorias em vigor (ou não se enquadram na grade nosológica científica) são negados, reinterpretados ou omitidos pelos biomédicos. Não chegam, assim, a ser construídos como fatos no cotidiano da atenção à saúde. Costumam ser reinterpretados como erros diagnósticos, evoluções anômalas, reações adversas ou idiossincráticas e sintomas psicossomáticos. Esse processo pode ser entendido como cegueira paradigmática (Tesser, Luz, 2002, p.367).

- Algumas ideias epistemológicas de Kuhn podem ajudar na compreensão dos processos de construção de saber e práticas dos médicos. Outra ordem de contribuições de Kuhn mais epistemológica e ética é a de que o reconhecimento dos paradigmas contribui para a tomada de consciência e o manejo das chamadas reduções paradigmáticas, o que melhoraria a habilidade clínica e o desempenho ético dos profissionais e alunos (Tesser, 2008, p.6).

- O ensino médico, por muito tempo, esteve centrado nos ambientes hospitalares e sob o domínio das especialidades médicas. Dessa forma, os valores, os exemplos e os conteúdos aprendidos vêm formando médicos pouco aptos a trabalharem com criatividade, satisfação e competência na atenção primária do Sistema Único de Saúde (SUS). Há poucos profissionais engajados e competentes para o trabalho na atenção primária e na Saúde da Família, relativamente nova e em construção, como de resto é o próprio SUS. Se a prioridade é a formação médica para a construção de um SUS melhor, a ambiência adequada para essa formação é o local onde ocorre, de fato, tal prática, ou seja, na própria rede básica e nas equipes da Estratégia de Saúde da Família (ESF) (Tesser, 2008, p.5).

\section{Considerações finais}

O problema que motivou a realização do presente trabalho foi a dificuldade dos profissionais da atenção básica de saúde no acolhimento ao sofrimento psíquico, percebida ao longo da minha prática 
como psiquiatra matriciadora. Supondo que essa dificuldade ocorresse mais por questões epistêmicas que por insuficiência de conteúdo, entendemos que uma revisão de literatura em publicações da área da saúde sobre o termo paradigma pudesse instrumentalizar essa discussão.

O atendimento ao indivíduo em sofrimento psíquico é apontado por esses profissionais como um dos desafios na rotina dos postos de saúde. As dificuldades apontadas são inúmeras, gerando as mais variadas reações e sentimentos nos profissionais, que, muitas vezes, negligenciam ou hostilizam o indivíduo em sofrimento. Na prática, o reflexo disso aparece no grande número de encaminhamentos para saúde mental e na tensão por vezes gerada nas discussões com o Núcleo de Apoio à Saúde da Família (NASF).

A chegada do NASF às unidades faz surgir a ideia de que discutir saúde mental na atenção básica aumentará o trabalho para as equipes já exauridas pelas exigências da população e dos gestores. Ao longo do trabalho, é possível se desconstruir essa ideia ao se discutir que a "cegueira paradigmática" não permite que o sofrimento psíquico seja construído como fato no cotidiano e, por isso, aparece como algo a mais a ser cuidado. Também é necessário se avançar na crítica de que os instrumentos disponibilizados pelo paradigma biomédico são insuficientes para o cuidado ao indivíduo em sofrimento psíquico e sua qualidade de vida.

Ao finalizar essa pesquisa, constatou-se que a discussão é atual e está avançada tanto na discussão teórica quanto da prática nos serviços de saúde e no ensino médico. O sofrimento psíquico pode ser considerado uma anomalia para o paradigma hegemônico, que não oferece recursos adequados aos profissionais de saúde da atenção básica para o acolhimento e abordagem dessa condição. A integralidade é um problema epistemológico para esse paradigma. A promoção da saúde aparece como um novo e promissor paradigma.

\section{Colaboradores}

Os autores Maria Luisa Vichi de Campos Faria e Ivan Amaral Guerrini participaram, igualmente, de todas as etapas de elaboração do artigo. 


\section{Referências}

ARAÚJO, E.C. Homeopatia: uma abordagem do sujeito no processo de adoecimento. Cienc. Saude Colet., v.13, supl., p.663-71, 2008.

ASSUNÇÃO, L.F.; MELO, G.C.M.P; MACIEL,D. Relação médico-paciente permeando o currículo na ótica do estudante. Rev. Bras. Educ. Med., v.32, n.3, p.383-89, 2008.

AYRES, J.R. Norma e formação: horizontes filosóficos para as práticas de avaliação no contexto da promoção da saúde. Cienc. Saude Colet., v.9, n.3, p.583-92, 2004.

BARABÁSI, A.-L.; GULBAHCE, N.; LOSCALZO, J. Network medicine: a network-based approach to human disease. Nat. Rev. Genet., v.12, p.56-69, 2011.

BARDIN, L. Análise de conteúdo. Lisboa: Edições 70, 1977.

BRASIL. Ministério da Saúde. Saúde mental e atenção básica: o vínculo e o diálogo necessários. Brasília: MS, 2003. (Circular conjunta n. 01/03, de 13/11/2003).

CAMPOS FARIA, M.L.V. Uma revisão bibliográfica sobre a utilização do termo paradigma em publicações científicas da área da saúde. 2009. Dissertação (Mestrado em Saúde Coletiva) - Faculdade de Medicina de Botucatu, Universidade Estadual Paulista Júlio de Mesquita Filho, Botucatu. 2009.

CARDOSO, M.H.C.A. História e medicina: a herança arcaica de um paradigma. Hist. Cienc. Saude - Manguinhos, v.6, n.3, p.551-75, 2000.

CLAUSET, A.; NEWMAN, M.E.J.; MOORE, C. Finding community structure in very large networks. Phys. Rev., v.70, n.6, p.1-6, 2004.

FORTES, S.; VILLANO, L.A.B.; LOPES, C.S. Perfil nosológico e prevalência de transtornos mentais comuns em pacientes atendidos em unidades do Programa de Saúde da Família (PSF) em Petrópolis, Rio de Janeiro. Rev. Bras. Psiquiatr., v.30, n.1, p.32-7, 2008.

FREITAS, C.M. Problemas ambientais, saúde coletiva e ciências sociais. Cienc. Saude Colet., v.8, n.1, p.137-50, 2003.

GALHARDI, W.M.P.; BARROS, N.F. O ensino da homeopatia e a prática no SUS. Interface - Comunic., Saude, Educ., v.12, n.25, p.247-66, 2008.

GUEDES, C.R.; NOGUEIRA, M.I.; CAMARGO Jr., K.R. A subjetividade como anomalia: contribuições epistemológicas para a crítica do modelo biomédico. Cienc. Saude Colet., v.11, n.4, p.1093-103, 2006.

GUIMARÃES, M.B.L. Condições de vida e pobreza: impasses e caminhos. In: CONGRESSO BRASILEIRO DE SOCIOLOGIA, 13., 2007, Recife. Anais ... Recife, 2007. Disponível em: <http://www.sbsociologia.com.br/portal/index.php?option= com_docman\&task = cat_view\&gid $=149 \&$ limit $=100 \&$ limitstart $=0 \&$ order $=$ hits \&dir $=$ ASC \&ltemid=171>. Acesso em: 12 abr. 2012.

JACOBINA, R.R. O paradigma da epistemologia histórica: a contribuição de Thomas Kuhn. Hist. Cienc. Saude - Manguinhos, v.6, n.3, p.609-30, 2000.

KUHN, T. A estrutura das revoluções científicas. São Paulo: Perspectiva, 1970.

LUZ, M.T. Cultura contemporânea e medicinas alternativas: novos paradigmas em saúde no fim do século XX. Physis, v.15, supl., p.145-76, 2005.

MARTINS, A. Novos paradigmas e saúde. Physis, v.9, n.1, p.83-112, 1999.

NOGUEIRA, M.I.; CAMARGO JR., K.R. A orientalização do Ocidente como superfície de emergência de novos paradigmas em saúde. Hist. Cienc. Saude - Manguinhos, v.14, n.3, p.841-61, 2007.

PAIM, J.S.; ALMEIDA FILHO, N. Saúde coletiva: uma "nova saúde pública" ou campo aberto a novos paradigma? Rev. Saude Publica, v.32, n.4, p.299-316, 1998. 
PEREIRA, M.A.O. et al. Saúde mental no Programa de Saúde da Família: conceitos dos agentes comunitários sobre o transtorno mental. Rev. Esc. Enferm. USP, v.41, n.4, p.567-72, 2007.

QUEIROZ, M.S. O itinerário rumo às medicinas alternativas: uma análise em representações sociais de profissionais da saúde. Cad. Saude Publica, v.16, n.2, p.363-75, 2000.

RIBEIRO, J.S. et al. Promoção da saúde e cultura cidadã envolvendo uma abordagem oftalmológica em escolares na Colônia Antônio Aleixo (CAA), Manaus-AM: uma experiência no ensino médico. Rev. Bras. Educ. Med., v.30, n.2, p.87-92, 2006.

SANTOS, M.E.S.B. Transtornos mentais comuns em pacientes com Aids que fazem uso de anti-retrovirais no Estado de São Paulo, Brasil. 2002. Dissertação (Mestrado) - Faculdade de Medicina, Universidade de São Paulo, São Paulo. 2002.

SOUSA, I.M.C.; VIEIRA, A.L.S. Serviços públicos de saúde e medicina alternativa. Cienc. Saude Colet., v.10, supl., p.255-66, 2005.

TESSER, C.D. Contribuições das epistemologias de Kuhn e Fleck para a reforma do ensino médico. Rev. Bras. Educ. Med., v.32, n.1, p.98-104, 2008.

TESSER, C.D.; LUZ, M.T. Uma introdução às contribuições da epistemologia contemporânea para a medicina. Cienc. Saude Colet., v.7, n.2, p.363-72, 2002.

VALLA, V.V. Globalização e saúde no Brasil: a busca da sobrevivência pelas classes populares via questão religiosa. In: VASCONCELOS, E.M. (Org.). A saúde nas palavras e nos gestos. São Paulo: Hucitec, 2001. p.39-62.

CAMPOS FARIA, M.L.V.; GUERRINI, I.A. Limitaciones del paradigma hegemónico científico en el acogimiento del sufrimiento psíquico en la atención primaria. Interface - Comunic., Saude, Educ., v.16, n.42, p.779-92, jul./set. 2012.

El problema que motivó este trabajo fue la dificultad para aceptar el sufrimiento psíquico en las unidades de atención primaria. Este trabajo investiga si esta dificultad se debe a la inadecuación epistemológica del paradigma hegemónico. Se realizó una revisión de la literatura y fue analizado el uso del término paradigma en la producción científica brasileña. Después de buscar las palabras paradigma y medicina y selección previa, combinadas con la selección anterior, 17 textos fueron seleccionados. El análisis se realizó en etapas: análisis linguístico y una revisión de los temas emergentes.

Determinamos las 50 palabras más frecuentes y las agrupamos en comunidades de coocurrencia con el algoritmo de Clauset. Los textosdiscutían las ideas de Kuhn frayendo experiencias innovadoras implementadas en unidades de salud y los cambios en los cursos de medicina. La promoción de la salud aparece como un paradigma prometedor para la salud.

Palabras clave: Paradigma. Medicina. Sufrimiento psíquico. Atención primaria de salud. Promoción de la salud. 